\title{
Cell transplantation therapies for spinal cord injury focusing on induced pluripotent stem cells
}

\author{
Masaya Nakamura ${ }^{1}$, Hideyuki Okano ${ }^{2}$ \\ ${ }^{I}$ Department of Orthopedics, School of Medicine, Keio University, 35 Shinanomachi, Shinjuku, Tokyo 1608582, Japan; ${ }^{2}$ Depart- \\ ment of Physiology, School of Medicine, Keio University, 35 Shinanomachi, Shinjuku, Tokyo 1608582, Japan
}

Stimulated by the 2012 Nobel Prize in Physiology or Medicine awarded for Shinya Yamanaka and Sir John Gurdon, there is an increasing interest in the induced pluripotent stem (iPS) cells and reprograming technologies in medical science. While iPS cells are expected to open a new era providing enormous opportunities in biomedical sciences in terms of cell therapies and regenerative medicine, safety-related concerns for iPS cell-based cell therapy should be resolved prior to the clinical application of iPS cells. In this review, the pre-clinical investigations of cell therapy for spinal cord injury (SCI) using neural stem/progenitor cells derived from iPS cells, and their safety issues in vivo, are outlined. We also wish to discuss the strategy for the first human trails of iPS cell-based cell therapy for SCI patients. Keywords: neural stem/progenitor cell; induced pluripotnet stem cell; spinal cord injury; transplantation Cell Research (2013) 23:70-80. doi:10.1038/cr.2012.171; published online 11 December 2012

\section{Introduction}

The central nervous system (CNS), including the brain and spinal cord, has been considered a representative example of organs in which regeneration is difficult. However, this commonly accepted theory is being disproved by recent progress in the field of stem cell biology. Neural stem/progenitor cells (NS/PCs) were experimentally identified by Reynolds and Weiss [1] in 1992, and subsequently, methods for culturing NS/PCs in mammals, including humans, have been established [1-5], which has allowed elucidation of the molecular biological characteristics of NS/PCs in the developmental process and in the CNS of adult mammals [6-10]. A number of studies have paid attention to the self-renewal capacity and multipotency of NS/PCs and tried to regenerate neural tissues lost as a result of neurodegenerative diseases and injuries. Particularly in the research field of spinal cord injury (SCI), mouse embryonic stem (ES) cell-derived NS/PCs [11] and rat embryonic spinal cord-derived NS/ PCs [12] have been transplanted into the injured spinal cord of rats, and human embryo-derived NS/PCs have

Correspondence: Hideyuki Okano

Tel: +81-3-5363-3746; Fax: +81-3-3357-5445

E-mail: hidokano@a2.keio.jp been transplanted into the injured spinal cord of the common marmoset in preclinical studies, aiming at clinical application [13, 14]. Safety of the cells and functional recovery were reported in all of the aforementioned studies. These results strongly suggest that application of In vitro passaged human NS/PCs for neural regeneration may be promising. However, clinical application has not yet been realized in Japan due to ethical issues with the use of NS/PCs derived from surplus embryos or aborted fetal tissues. In fact, the revised version of the Japanese Ministry of Health, Labor and Welfare's Guidelines for Clinical Research Using Human Stem Cells was launched on 1 November 2010. That appears to be a major step in terms of the future development of regenerative medicine in Japan. However, fetal and ES cellderived NS/PCs are still not covered by the guidelines.

Under such circumstances, Yamanaka et al. [15-17] have introduced several genes into somatic cells to create induced pluripotent stem (iPS) cells with ES cell-like pluripotency and proliferative capacity. If adult somatic cells, such as blood cells or skin fibroblasts, can be converted into NS/PCs via iPS cells for transplantation into injured spinal cord, the aforementioned problems, such as ethical issues and rejection in transplantation, could be circumvented. This review outlines the history of research using fetal-derived NS/PCs, In vitro methods for inducing the differentiation of ES and iPS cells 
into NS/PCs that can possibly serve as the cell sources in place of fetal-derived NS/PCs, and furthermore, the safety of transplantation of iPS cell-derived NS/PCs and of their transplantation into SCI models.

\section{Regenerative medicine for SCI using fetal-derived NS/PCs}

Transplantation of in vitro propagated NS/PCs into the injured spinal cord began to be studied, since previous studies demonstrated the efficacy of transplantation of a fetal spinal cord tissue in a rat model of SCI. However, unfortunately, its efficacy could not be appreciated at first. It was considered that when NS/PCs are transplanted immediately after injury, the transplanted NS/PCs mostly differentiate into astrocytes and do not contribute to functional recovery of the injured spinal cord [18]. Ogawa et al. [12] reported that the transplantation of rat fetal spinal cord-derived NS/PCs into a rat model of cervical spinal cord contusion injury 9 days after injury resulted in significant functional recovery of the upper extremities as compared with that in the control group. It was revealed that the grafted cells differentiated into neurons, astrocytes and oligodendrocytes, and that in particular, the transplanted cell-derived neurons formed functional synapses with the host neurons. These results suggest that allowance of a therapeutic time window after injury is important before transplantation of $\mathrm{NS} /$ PCs into the injured spinal cord. Namely, the acute phase of SCI corresponds to the "inflammatory phase" due to the upregulation of inflammatory cytokines, excitatory neurotransmitters and free radicals, and is not suitable for transplantation, whereas in the chronic phase, about 2 weeks or more after injury, the injury enters the stage of glial scar formation, which prevents axonal regeneration. Therefore, the subacute phase of SCI is considered as the optimal time window for NS/PC transplantation in a rat SCI model [10, 12, 19] (Figure 1). However, we have to realize that the anatomy and functions of the spinal cord are considerably different between rodents and primates. Thus, it is very important to test proof of concept on the effectiveness of fetal NS/PCs transplantation for SCI in non-human primates. For this purpose, Iwanami et al. [13, 14] established a SCI model of a non-human primate, the common marmoset (Callithrix jacchus), and transplanted human aborted fetal forebrain-derived NS/PCs into this

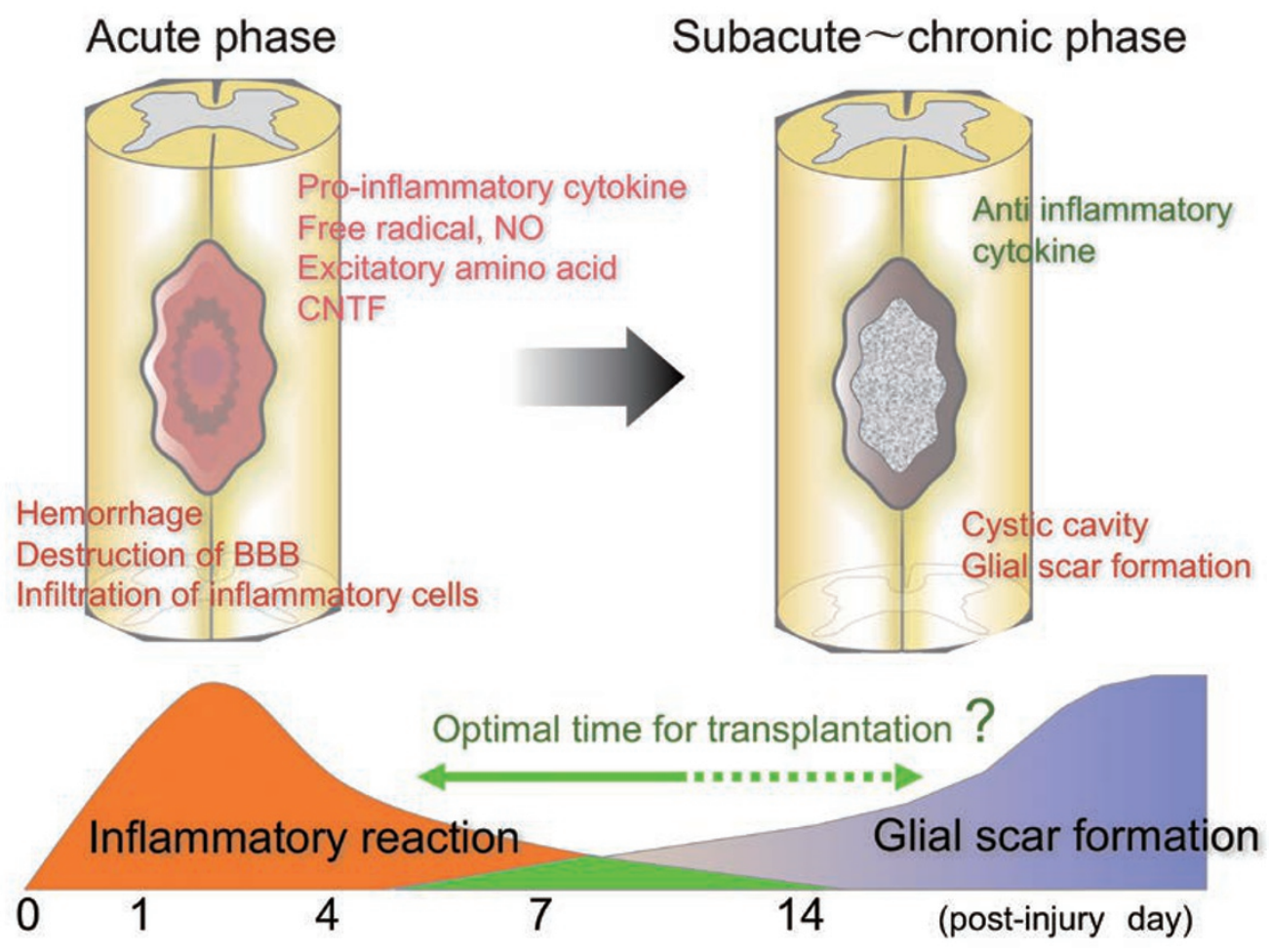

Figure 1 Microenvironment of the injured spinal cord. Because the immediately post-traumatic microenvironment of the spinal cord is in an acute inflammatory stage, it is not favorable for the survival and differentiation of NS/PC transplants. On the other hand, in the chronic stage after injury, glial scars form in the injured site that inhibit the regeneration of neuronal axons. Thus, we believe that the optimal timing of transplantation is 1-2 weeks after injury. 
model. The transplanted cells survived and differentiated into neurons, astrocytes and oligodendrocytes, thereby promoting functional recovery as compared with that in the control group, and also no tumorigenesis from the transplanted cells was observed during the observation period (3 months after transplantation) [13]. Reconstruction of neural circuits by synapse formation between the transplanted cells and host neurons, and remyelination and trophic support provided by the transplanted cells are considered as being among the important mechanisms of the motor function recovery [20]. These results suggest that fetal tissue-derived NS/PCs are very useful as cell sources for transplantation. However, as described above, clinical application of these cells has not yet been realized in Japan due to the ethical issues associated with the use of aborted fetal tissues.

\section{ES cell-derived NS/PCs}

In vitro models of neural development and mouse ES cell-derived NS/PCs

NS/PCs are defined as cells that have self-renewal capacity and multipotency. However, their differentiation and proliferative capacity is strictly regulated according to the stage at which these cells are produced and their localization, and not all NS/PCs have identical characteristics. NS/PCs are known to already exist around embryonic day 5 (E5.5), and the NS/PCs at this stage can be cultured in the presence of leukemia inhibitory factor (LIF) [21]. On embryonic days E8.5-12.5, NS/PCs can be cultured In vitro in the presence of fibroblast growth factor-2 (FGF-2). From this stage to the late embryonic stages, radial glial cells around the ventricles can serve as NS/PCs, and these cells self-renew by symmetric divisions and generate neurons by asymmetric divisions [22, 23]. From the late developmental to the neonatal stage and in the adult brain, NS/PCs exist mainly around the ventricles, and differentiate not only into neurons but also into glial cells (astrocytes and oligodendrocytes) [24]. During and after the late embryonic stages, NS/PCs are stimulated to proliferate not only by FGF-2 but also by epidermal growth factor (EGF). However, the NS/PCs that appear during and after the late embryonic stages cannot generate early-born projection neurons, such as forebrain cholinergic neurons, dopaminergic neurons and motor neurons. Okada et al. [25] induced the development of highly plastic NS/PCs existing at relatively early developmental stages using mouse inner cell massderived ES cells, and succeeded in constructing a culture system mimicking the temporal and spatial specificity of the developmental process (Figure 2). In this culture system, first LIF, which is required to maintain the undif- ferentiated state, is removed and ES cells are cultured in suspension to induce the formation of embryoid bodies (EBs) containing cells derived from the three germ layers. These EBs contain relatively early-stage NS/PCs, which can be selectively cultured as neurospheres by suspended culture in serum-free medium for NS/PCs in the presence of FGF-2. Furthermore, the addition of Noggin, which inhibits BMP to promote differentiation into the neuroepithelium, thereby playing an important role in forebrain formation, or retinoic acid (RA), which is known to play an important role in neural induction and also in the development of the hindbrain and anterior spinal cord, at a low concentration during EB formation increased the proportion of NS/PCs in the EBs and increased the efficiency of neurosphere formation. Primary neurospheres thus formed can be passaged to generate secondary and tertiary neurospheres. Interestingly, almost only neurons are induced from primary neurospheres, whereas not only neurons but also glial cells, such as astrocytes and oligodendrocytes, are induced from secondary and tertiary neurospheres. These mouse ES cell-derived neurospheres (ES-NS) can be repeatedly passaged and have the ability to generate neurons, astrocytes and oligodendrocytes, indicating that these neurospheres contain NS/PCs with self-renewal capacity and multipotency. In addition, such a change in the differentiation capacity with culture passage well reflects the developmental process of the CNS, in that only neurons are generated in the early developmental stages, whereas glial cells are generated for the first time during and after the mid-gestation stages. Furthermore, the regional specificity of the induced NS/PCs along the anteroposterior axis can be regulated by changing the concentration of Noggin and RA added during EB formation. Moreover, the regional specificity along the dorsoventral axis has also been successfully regulated by the addition of the ventralizing factor, Sonic Hedgehog, and dorsalizing factors, BMP4 and Wnt3a, during the formation of the primary neurospheres [25]. These results suggest that the regional specificity of NS/PCs could be regulated by the addition of appropriate factors at appropriate stages during induction of differentiation.

Transplantation of mouse ES cell-derived NS/PCs into the injured spinal cord

Methods of inducing mouse ES cells to differentiate into neurons have been widely studied [26], and ES cellderived NS/PCs (ES-NS/PCs) are ideal cell sources for transplantation. The differentiation stages at which ESNS/PCs are transplanted are variable, and could range from undifferentiated ES cells to EBs and differentiated neurons. However, it is known that the lower the degree 


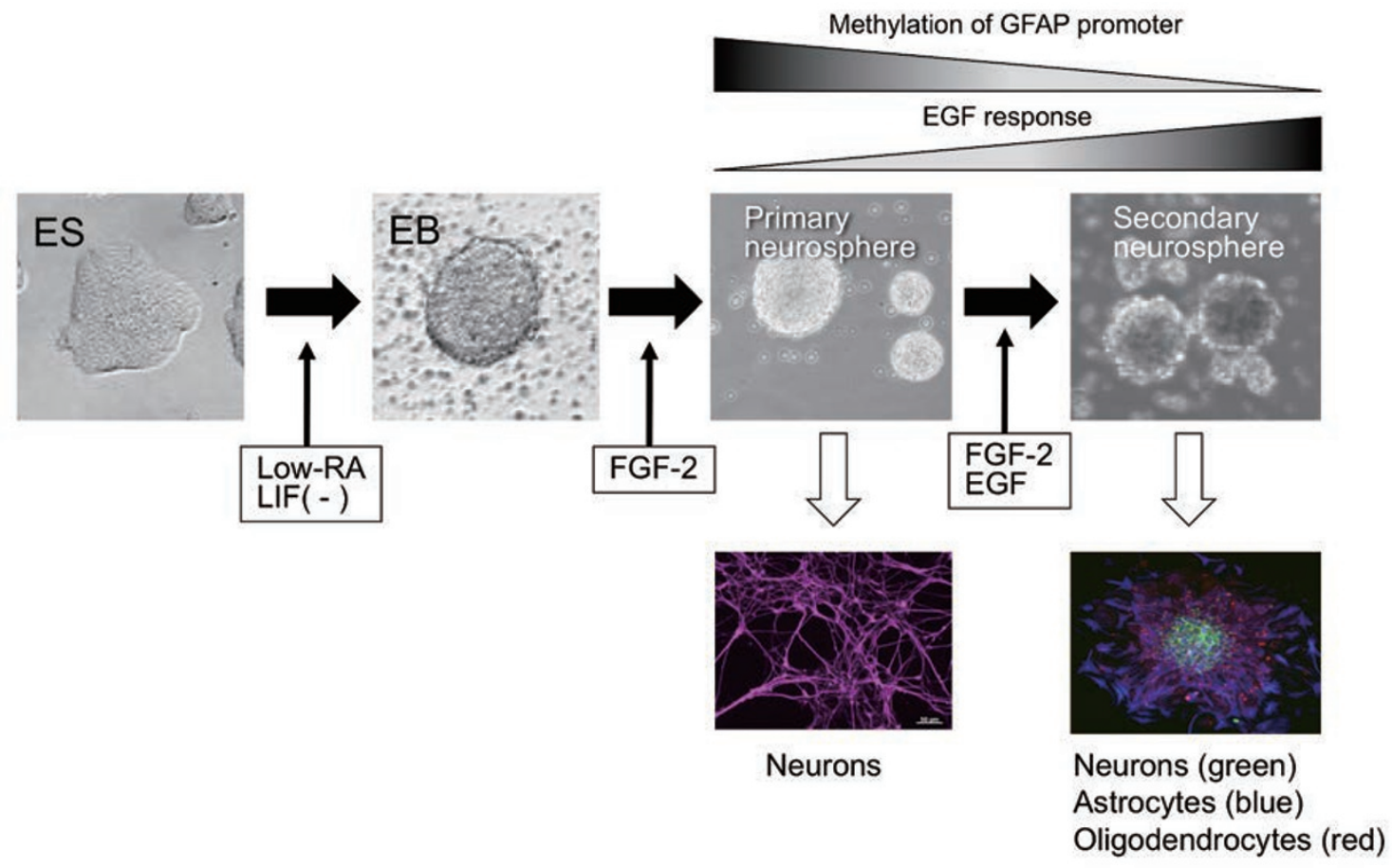

Figure 2 Neural induction of mouse ES cells through EBs. After the removal of LIF, ES cells are cultured in suspension to induce the formation of EBs. EBs contain relatively early-stage NS/PCs, which can be selectively cultured as neurospheres by suspended culture in serum-free medium for NS/PCs in the presence of FGF-2. Primary neurospheres can be passaged to generate secondary and tertiary neurospheres. Interestingly, almost only neurons are induced from primary neurospheres, whereas not only neurons but also glial cells, such as astrocytes and oligodendrocytes, are induced from secondary and tertiary neurospheres.

of differentiation, the higher the incidence of teratomas derived from the transplanted cells [27]. As regards the efficacy of ES-NS/PC transplantation into the injured spinal cord, McDonald et al. [11] demonstrated that the transplantation of EBs generated from mouse ES cells into the injured spinal cord of rats resulted in good functional recovery. However, the risk of tumorigenesis in the long term associated with transplantation of EBs at lesser stages of differentiation cannot be ignored. Keirstead et al. [28] established an effective method of inducing human ES cells to differentiate into highly pure populations of oligodendrocyte progenitors by using a culture medium containing factors promoting differentiation into oligodendrocytes, such as insulin and thyroid hormones, and reported that the transplantation of these oligodendrocyte progenitors into the injured spinal cord of rats resulted in remyelination of demyelinated axons and recovery of motor function. Subsequently, Yamada et al. [29] used electrically stimulated mouse EBs generated from ES cells to induce selective differentiation into neurons for transplantation into the injured spinal cord. They found that while the transplanted EBs differenti- ated into neurons in the injured spinal cord at a higher frequency than the transplanted EBs without electrical stimulation, the proliferative capacity of the electrically stimulated EBs was lower than that of the non-stimulated EBs. Thus, this method was effective from the aspect of safety, but did not lead to satisfactory recovery of motor function after SCI [29]. None of these studies has clarified exactly which stage of induction from ES cells to NS/PCs contained in the neurospheres would be the most suitable for transplantation therapy at the subacute phase of SCI.

To determine this issue, Kumagai et al. [30] transplanted primary neurospheres induced from mouse ES cells via EB formation using the above-described culture system, and secondary neurospheres, obtained after one passage, into a mouse SCI model to evaluate their efficacy. As described above, most of the primary neurospheres differentiated into neurons, whereas the secondary neurospheres differentiated into three types of cells, astrocytes, oligodendrocytes and neurons [25]. The engraftment rate was $\sim 20 \%$ for both transplanted primary and secondary neurospheres. It is of interest that primary 
neurospheres showed neuron-dominant differentiation, whereas the secondary neurospheres differentiated into neurons and glial cells, consistent with their In vitro characteristics. The transplantation of secondary neurospheres significantly prevented the atrophy and demyelination of the injured spinal crod and enhanced the axonal regrowth and angiogenesis as compared with the primary neurospheres transplantation group. Interestingly, behavioral analysis using the Basso Mouse Scale (BMS) revealed that only the secondary neurospheres transplantation group showed statistically significant functional recovery as compared to the vehicle control group. These results suggest that in the application of ES-derived NS/ PCs to the treatment of SCI, it would be more desirable to transplant NS/PCs generating both neurons and glial cells than to transplant those differentiating dominantly into neurons.

However, as in the case of embryo-derived NS/PCs, ethical issues need to be confronted before attempts at clinical application of ES-derived NS/PCs in the future, because surplus embryos are used for establishing human ES cells.

\section{iPS cell-derived neurospheres}

Safety assessment of mouse iPS cell-derived neurospheres

iPS cells established by Yamanaka et al. have paved the way for the development of solutions for the abovementioned ethical issues related to the use of embryos and aborted tissues $[15,16]$. iPS cells are pluripotent stem cells that are generated by introducing Oct4, Sox2, Klf4 and $c-M y c$ genes into mouse/human fibroblasts to reprogram somatic cells, and exhibit proliferative and differentiation capacity almost equivalent to that of ES cells. iPS cells are expected to be a solution to problems such as the ethical issues and immunological rejection, because these cells can be established from the somatic cells of each patient. On the other hand, iPS cells may be associated with a greater risk of tumorigenesis than ES cells, because (1) foreign genes are introduced into the chromosome, and (2) there is the possibility that the reprograming is not necessarily complete. Miura et al. [31] have revealed that the responsiveness of mouse iPS cells to induction of neural differentiation and their safety after transplantation vary greatly depending on the somatic cells from which the iPS cells are derived. Thirty-six mouse iPS cell lines established in our laboratory were induced to differentiate into neural lineages and transplanted into the striata of the brains of immunodeficient NOD/severe combined immunodeficiency (SCID) mice as neurospheres to evaluate their In vivo differentiation capacity and safety after transplantation. All of the iPS cell line-derived neurospheres (iPS-neurospheres) were analyzed in detail by flow cytometry, and it was found that the proportion of Nanog-EGFP-positive undifferentiated cells remaining in the neurospheres varied greatly depending on the type of somatic cells from which the iPS cells were derived [31]. Mouse embryonic fibroblast (MEF)-derived iPS-neurospheres showed responsiveness to differentiation induction equivalent to that of ES cells, and almost no undifferentiated cells remained in the neurospheres. The frequency of post-transplantation teratoma formation in the MEF-iPS-neurospheretransplanted mouse groups was as low as that in the ESneurosphere-transplanted group. No teratoma formation was observed during the 16-week observation period in the groups transplanted with neurospheres induced from either of the two iPS cell lines established from adult gastric epithelial cells. On the other hand, adult tail tip fibroblast (TTF)-derived iPS cell lines showed significant resistance to differentiation, so that many undifferentiated cells remained in the neurospheres after the induction of differentiation. Teratoma formation was observed at a high frequency in the mouse groups transplanted with these neurospheres, and many mice became weak or died within a short time. The responsiveness of adult hepatocyte (Hep)-derived iPS cell lines to differentiation induction and their tumorigenicity were intermediate between those of the MEF-iPS cell lines and TTF-iPS cell lines [31]. Introduction/non-introduction of c-Myc and selection/non-selection of reprogramed cells by reporters at the time of establishment of the cell line did not affect the responsiveness of the iPS cells to differentiation induction or their safety after transplantation. The differences in the differentiation capacity of iPS cells depending on the somatic cells from which the iPS cells were derived may be caused by the epigenetic memory, i.e., the profiles of the remaining expressed genes from the somatic cells, and it is an urgent future task to further analyze the nature of these cells in greater detail.

\section{Treatment of SCI using "safe" mouse iPS cell clone- derived secondary neurospheres}

Secondary neurospheres were induced from the mouse iPS cells (clone 38C2) established from the MEF (hereafter referred to as 38C2-iPS-secondary neurospheres) and transplanted into a mouse SCI model, after their safety was confirmed by the above-described transplantation experiments into immunodeficient mouse brain [32] Contusion SCI was induced at T10 level, and 9 days after the injury, 38C2-iPS-secondary neurospheres were transplanted into the injured spinal cord. The gene for the luminescent enzyme luciferase, CBRluc, and a red 
fluorescent protein gene, $m R F P$, were introduced into the cells to be transplanted using a lentivirus, and the survival of the transplanted cells was sequentially monitored by bioimaging [33] for 6 weeks after the injury. Thereafter, quantitative assessment using bioimaging revealed that $\sim 20 \%$ of the transplanted cells were engrafted with no apparent increase in the amount of luminescence, and histological analysis revealed no tumor formation up to at least 5 weeks after the transplantation (Figure 3). The transplanted cells differentiated into Hu-positive neurons, GFAP-positive astrocytes and GST- $\pi$-positive oligodendrocytes, at efficiencies of $\sim 30 \%, 50 \%$ and $15 \%$, respectively. Behavioral analysis using the BMS revealed that the 38C2-iPS-secondary neurospheres transplantation group showed functional recovery almost equivalent to that observed in the mouse ES-secondary neurospheres transplantation group, and significantly greater recovery of hindlimb motor function as compared to the vehicle control group injected with culture medium alone (Figure 4A). Analysis of the mechanism of this functional recovery revealed that the transplanted $38 \mathrm{C} 2$-iPS-secondary neurospheres differentiated into MBP-positive mature oligodendrocytes, which remyelinated the nerve fibers demyelinated by the injury (Figure 4B). As a result, the myelin sheath area, which was positively stained with Luxol fast blue (LFB), significantly increased in the 38C2-iPS-secondary neurospheres transplantation group as compared with that in the vehicle control group (Figure $4 \mathrm{C}$ and $4 \mathrm{D})$. Furthermore, it is possible that the transplanted cells differentiated into immature astrocytes with bipolar processes in the injured spinal cord and that these immature astrocytes played a role in the guidance of
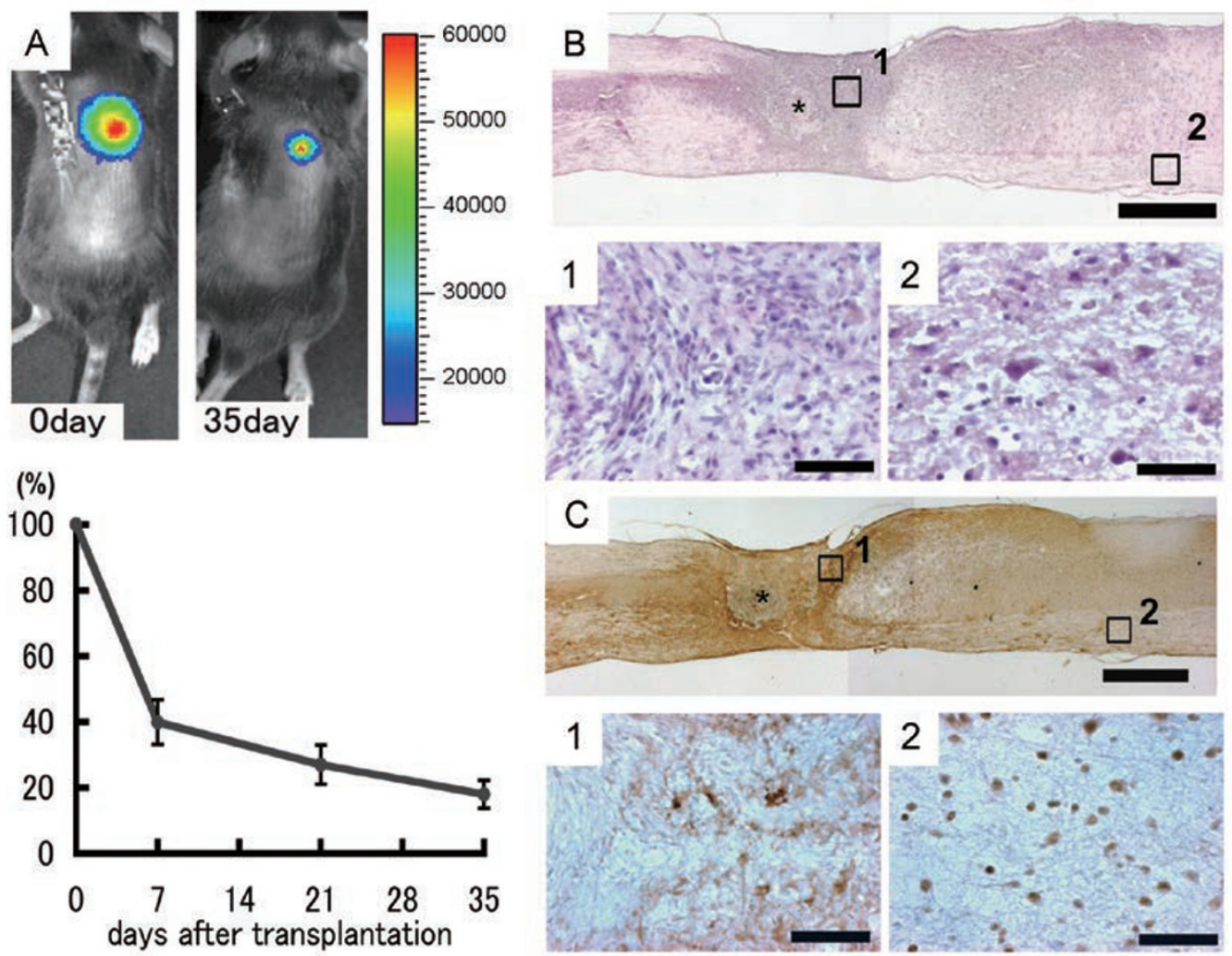

Figure 3 Transplanted safe 38C2-iPS-secondary neurospheres survive without any evidence of tumorigenesis [32]. (A) Representative BLI images of a mouse in which CBRluc-expressing 38C2-iPS secondary neurospheres were transplanted into the injured spinal cord (left, immediately after transplantation; right, 42 days after transplantation). Quantification of the photon intensity revealed that $\sim 60 \%$ of the grafted cells were lost within 7 days after transplantation, and $\sim 20 \%$ of the cells survived 35 days after transplantation. (B) HE and (C) anti-RFP DAB staining of sagittal sections of the spinal cord 42 days after injury (38C2-iPS-secondary neurospheres transplanted). There was no evidence of tumorigenesis (B). No significant nuclear atypia was observed in magnified images of the boxed areas showing the lesion epicenter (B-1) or white matter caudal to the transplantation site (B-2). Grafted cells survived and were diffusely distributed rostral and caudal to the lesion site (C). Higher-magnification images of the boxed areas showing the lesion site $(\mathbf{C}-\mathbf{1})$ and white matter caudal to the lesion site $(\mathbf{C}-\mathbf{2})$. *Lesion epicenter. Images are reproduced from reference [32]. 

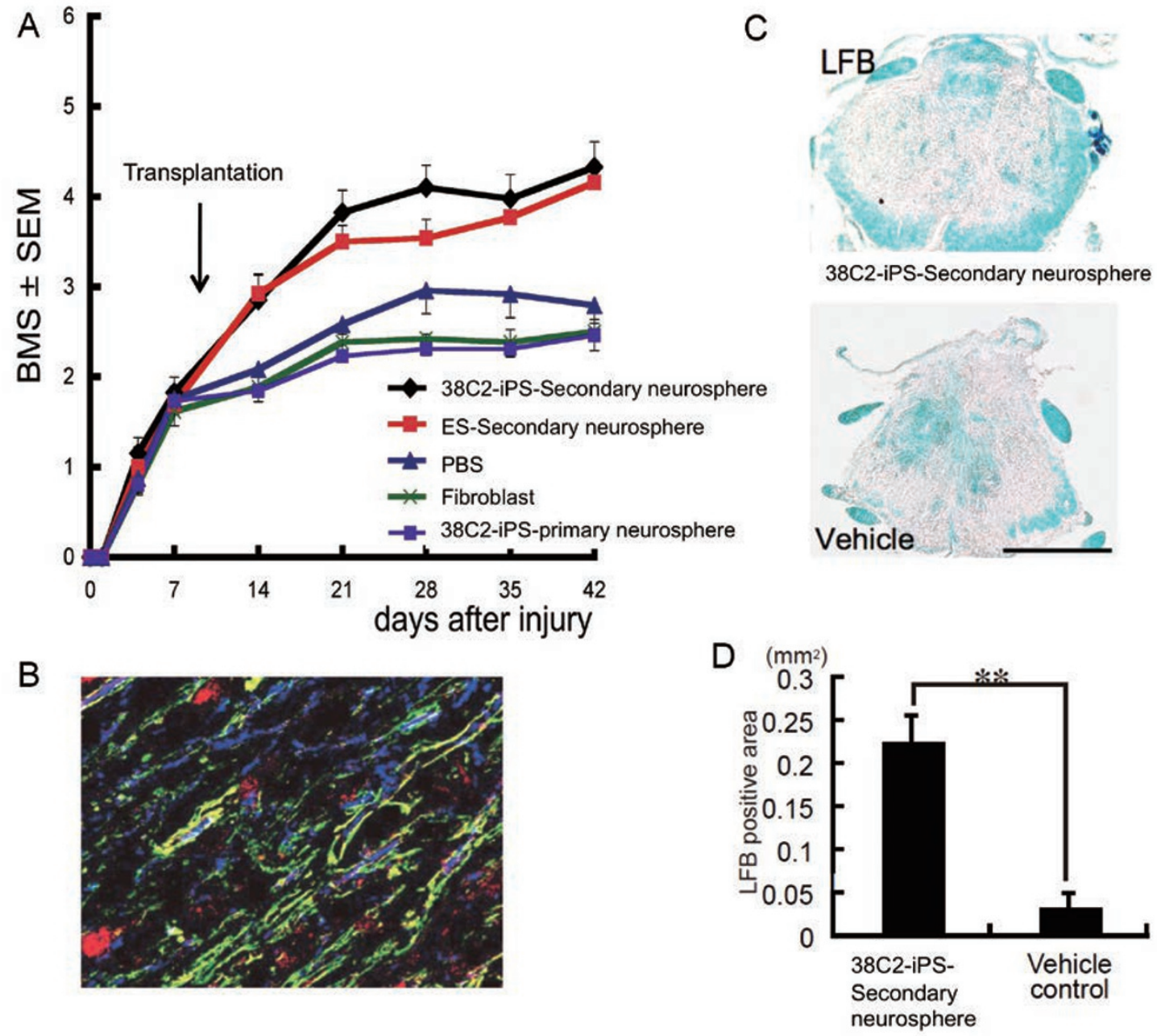

Figure 4 Transplanted secondary neurospheres derived from safe MEF-iPS clones contributed to the remyelination, thereby promoting functional recovery [32]. (A) 38C2-iPS-derived secondary neurosphere-transplanted mice showed significantly better functional recovery compared to the PBS and fibroblast control mice. (B) Immunohistochemistry of grafted secondary neurosphere-derived mature oligodendrocytes (MBP, green; RFP, red and NF200kD, blue). Grafted cells were integrated into myelin sheath (yellow). (C) LFB staining of axial sections of the spinal cord at the lesion epicenter 42 days after injury; 38C2iPS-derived secondary neurospheres transplanted and vehicle control animals. (D) Quantification of LFB-positive areas at the lesion epicenter 42 days after injury $\left.{ }^{* *} P<0.01\right)$. Images are reproduced from reference [32].

regenerating axons. In fact, 5-HT-positive raphe-spinal fibers, which are considered to play a great role in motor function in rodents, have been reported to be present in large numbers in the vicinity of these immature astrocytes, and quantification of these 5-HT-positive fibers at a distance of $4 \mathrm{~mm}$ from the site of injury revealed a significant increase of their number in the transplantation group (Figure 5). These results indicate that remyelination and glial support for the raphe-spinal fibers provided by the transplanted cells are the major mechanisms of recovery of the hindlimb function by 38C2-iPS-secondary neurospheres transplantation [32].
"Safe" and "dangerous" mouse iPS cell clones derived from adult tissues

Next, we performed similar transplantation experiments using adult tissue (TTF)-derived iPS cells, aiming at clinical application in autologous cell transplantation. Of the 36 mouse iPS cell clones used in the abovedescribed safety assessment, 6 clones were derived from TTF, of which, only the 335D1 clone was confirmed to be safe [31]. This 335D1 clone and two TTF-derived "dangerous" clones with tumorigenicity $(256 \mathrm{H} 13$ and $256 \mathrm{H} 18$ ) were induced to form neurospheres (335D1iPS-secondary neurospheres, 256H13-iPS-secondary neurospheres and 256H18-iPS-secondary neurospheres, 


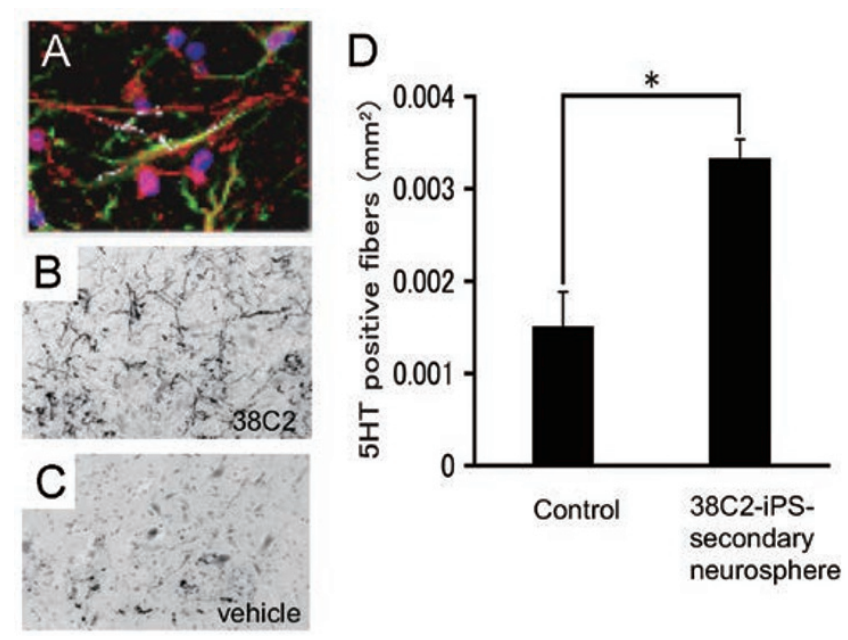

Figure 5 Transplantation of safe MEF-iPS-secondary neurospheres promoted serotonergic innervation of the dorsal cord [32]. (A) Immunohistochemistry of 38C2-iPS-secondary neurosphere-derived astrocytes closely associated with $5 \mathrm{HT}+$ serotonergic fibers. (RFP, red; GFAP, green and 5-HT, white) (B, C) Transplantation of 38C2-iPS-derived secondary neurospheres promoted the growth of $5 \mathrm{HT}+$ serotonergic fibers in the distal spinal cord. Axial sections of 38C2-iPS-derived secondary neurospheres transplanted (B) and vehicle control mice (C). (D) Quantitative analysis of $5 \mathrm{HT}+$ serotonergic fibers of distal cord in the vehicle control and 38C2-iPS-derived secondary neurospheres transplantation groups ( 6 weeks post injury) $\left({ }^{*} P<0.01\right)$. Images are reproduced from reference [32]. respectively) and transplanted into a mouse SCI model. Although functional recovery was obtained in all groups transplanted with these neurospheres, the recovery was transient and was suddenly lost about 6 weeks after the injury, and the majority of the mice subsequently died in both the 256H13- and 256H18-iPS-secondary neurospheres transplantation groups due to the formation of teratomas. In contrast, in the 335D1-iPS-secondary neurospheres transplantation group, no tumor formation was observed in any of the mice, and the functional recovery was significantly better than that in the vehicle control group and equivalent to that in the ES-secondary neurospheres transplantation group. These results indicate that adult tissue-derived iPS cell clones could serve as useful cell sources for the treatment of SCI provided that their safety is strictly evaluated in advance [32].

\section{Treatment of SCI using human iPS cell-derived neuro- spheres}

Aiming for the eventual clinical application, we moved on to the transplantation of human iPS-neurospheres into injured spinal cord of NOD-SCID mice. Oct4, Sox2, Klf4 and $c-M y c$ were introduced into adult facial skin-derived fibroblasts using a retrovirus to create the cell line 201B7 [15], which was used as the cell source. The 201B7 clone was induced to differentiate into neurospheres (201B7iPS-neurospheres) using a method similar to that used for
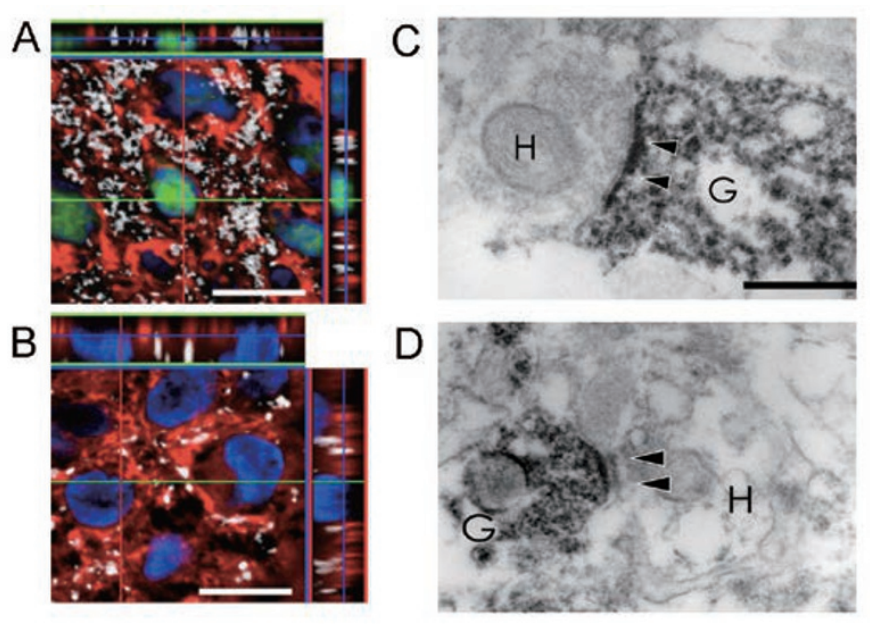

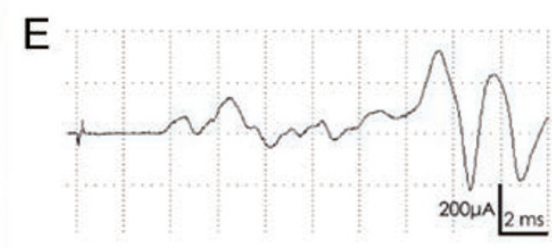

hiPS cell derived neurosphere

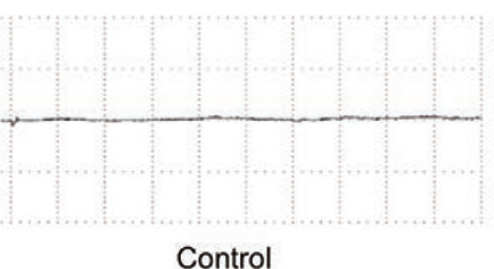

Figure 6 Evidence for synapse formation between human iPS cell (201B7)-derived neurons and host mouse spinal cord neurons [34]. (A) Sections were triple-stained with HNu (green), $\beta I I I$ tubulin (red) and the presynaptic marker Bassoon (Bsn, white). The Bsn antibody used here recognized the rat and mouse, but not human, protein. (B) Sections triple-stained for $\mathrm{HNu}$ (blue), $\beta$ III tubulin (red) and the human-specific presynaptic marker hSyn (white). (C, D) Electron microscopy showing synapse formation between host mouse neurons and graft-derived Venus+ (black) human neurons: the pre- and postsynaptic structures indicated transmission from a host neuron to a graft-derived neuron (C) and from a graft-derived neuron to a host neuron (D). H, host neuron; G, graft-derived neuron; arrowheads, postsynaptic density. (E) Electrophysiological analysis performed 112 days after SCI. MEP waves were detected in most of the transplantation group (14 out of 17), whereas they were not detected in the control group (0 out of 15). Images are reproduced from reference [34]. 
mouse iPS cells. The safety of 201B7-iPS-neurospheres has already been confirmed by our analyses. 201B7-iPSneurospheres were transplanted into the injured spinal cord of NOD-SCID mice to evaluate their therapeutic effect [34]. The transplanted cells were well engrafted in the mouse spinal cord and differentiated into NeuN- and $\beta I I I$ tubulin-positive neurons, GFAP-positive astrocytes and APC-positive oligodendrocytes. Approximately 50\% of the transplanted cells differentiated into $\beta \mathrm{III}$ tubulinpositive neurons, and of these, $\sim 70 \%$ differentiated into GAD67-positive $\gamma$-aminobutyric acid (GABA)-ergic neurons. In addition, immunohistochemical and electronmicroscopic analyses confirmed that the neurons derived from the transplanted cells formed synapses with the host neurons (Figure 6A-6D). Motor evoked potential (MEP) waveforms could be detected in the 201B7-iPSneurospheres transplantation group, but not in the vehicle control group, suggesting that the neurons derived from the transplanted cells functioned as interneurons in the mouse spinal cord, contributing to the reconstruction of neural circuits (Figure 6E and 6F). Furthermore, angiogenesis, nerve regeneration and tissue protection, which were likely to be mediated by paracrine actions of graftderived astrocytes, were also observed. BMS, rotarod test and gait analysis using a treadmill showed good improvement in the lower extremity motor function in the 201B7-iPS-neurospheres transplantation group (Figure 7). Furthermore, to confirm the long-term safety of 201B7-iPS-neurospheres transplantation, follow-up was continued for $\sim 4$ months after the SCI, which revealed that the functional recovery was maintained without tumor formation [34]. Consistently, Fujimoto et al. [35] also confirmed the efficacy and safety of human iPS-NS/ PC transplantation for SCI treatment in immunodeficient mice.

Based on these findings, we moved on to 201B7neurospheres transplantation for treatment of SCI in common marmosets as previously reported $[13,14]$. Grafted hiPS-neurospheres survived and differentiated into NeuN-positive neurons, GFAP-positive astrocytes and Olig1-positive oligodendrocyte progenitor cells. hiPS-neurospheres transplantation enhanced axonal regrowth, myelination and angiogenesis, thereby promoting functional recovery after SCI. It was noteworthy that there was no tumor formation at least for 12 weeks after transplantation [36]. Taken together, pre-evaluated safe hiPSC-derived neurospheres could be a potential cell source for SCI treatment in clinic.

\section{Future problems and perspective}

As described above, iPS cells could offer great prom-
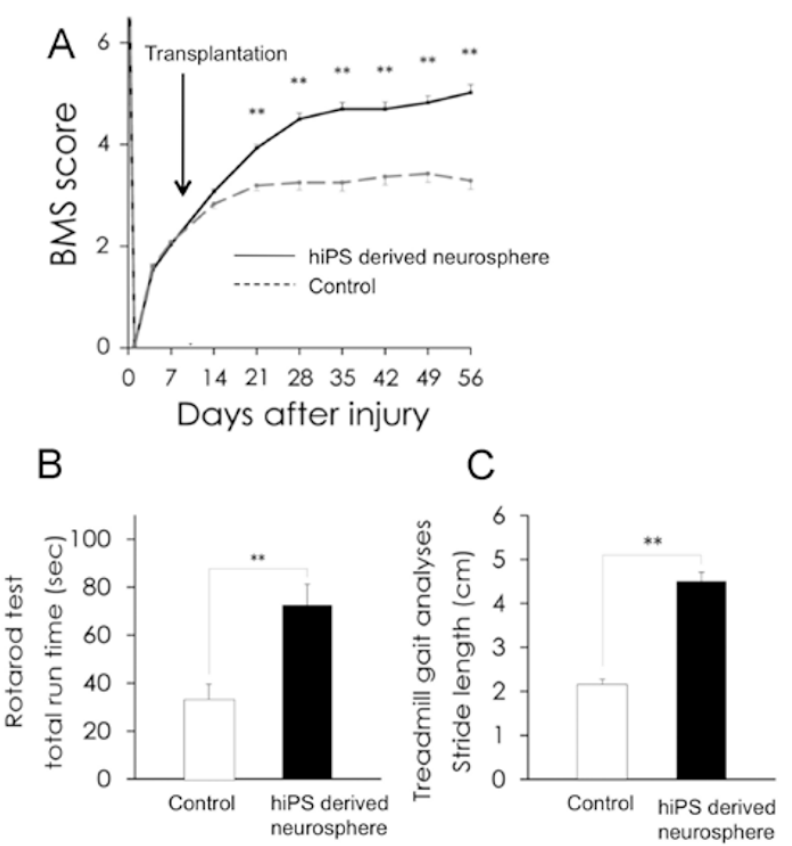

Figure 7 Transplanted human iPS cell (201B7)-derived neurospheres promoted motor functional recovery after SCI [34]. (A) Motor function in the hindlimbs was assessed weekly by the BMS score for 56 days. Values are means \pm SEM. (B) Rotarod test 56 days after $\mathrm{SCl}$. Graph shows the total run time. Values are means \pm SEM. (C) Treadmill gait analysis using the DigiGait system 56 days after SCl. Graph shows stride length. Values are means \pm SEM. $\left({ }^{\star \star} P<0.01\right)$. Images are reproduced from reference [34].

ise as the cell source for autologous transplantation. Recently, it has become possible to establish human iPS cells from skin fibroblasts and also drops of blood $[15,37,38]$; thus, research on iPS cells has been rapidly advancing. However, retroviruses and lentiviruses are commonly used when reprograming factors are introduced in the establishment of iPS cells; these viruses are often integrated near the gene promoters, increasing the risk of tumorigenesis by changing the expression of endogenous genes in the vicinity. In fact, according to one study, 2 out of 10 patients with X-linked SCID (X-SCID) who received gene therapy with a retroviral vector developed leukemia [39]. Recently, research on this problem has rapidly progressed, and many studies have proposed solutions [40], including establishment of iPS cells with transient gene expression instead of using retroviruses or lentiviruses [38, 41-43], by introducing proteins [44$46]$, by substituting some genes with drugs $[44,46]$ and by using minicircle vectors that enable longer-term gene expression than plasmid vectors [47]. According to the results of our previous studies, transgene reactivation 
and incomplete reprograming are considered as the main causes of tumorigenesis, and we propose to use integration-free iPS cells reprogramed using episomal vectors in the future to overcome the first of these problems [40, 48]. Furthermore, among our most important tasks before successful clinical application will be to induce Glis-1transduced iPS cells, developed by Yamanaka et al. [49], which are reprogramed more completely, this allowing them to differentiate reliably into NS/PCs, and to accurately evaluate the safety of this final product. In addition to these improvements of the iPS cells, the safety issues must be validated through the intensive quality examination of iPS cell-derived NS/PCs in terms of genetic and epigenetic status, and their differentiation, proliferation and tumorigenicity In vivo, prior to the first human trials [40]. Finally, another important challenge before attempting clinical application pertains to the use of agents and cells derived from xenogeneic sources. Currently, animal-derived serum (bovine serum) is used for establishing iPS cells and inducing neural differentiation; however, a method to establish iPS cells without using such serum has also been reported [50], although these iPS cells will have to be characterized from the beginning. To accelerate these preclinical studies in the future, traceability of animal-derived agents should be ensured and methods of clean-up at the stage of the final product, namely, iPS-NS/PCs, according to GMP should be established as a practical strategy.

\section{Acknowledgments}

The original investigation on iPS cells was performed in collaboration with Prof Shinya Yamanaka (Center for iPS Cell Research and Application, Kyoto University, Japan). This work was supported by the Japan Science and Technology-California Institute for Regenerative Medicine collaborative program, grants from Grants-in-Aid for Scientific Research from JSPS and the Ministry of Education, Culture, Sports, Science and Technology of Japan (MEXT), the project for realization of regenerative medicine and support for the core institutes for iPS cell research from MEXT and by a Grant-in-aid for the Global COE program from MEXT to Keio University.

\section{References}

1 Reynolds BA, Weiss S. Generation of neurons and astrocytes from isolated cells of the adult mammalian central nervous system. Science 1992; 255:1707-1710.

2 Davis AA, Temple S. A self-renewing multipotential stem cell in embryonic rat cerebral cortex. Nature 1994; 372:263-266.

3 Fernandes KJ, McKenzie IA, Mill P, et al. A dermal niche for multipotent adult skin-derived precursor cells. Nat Cell Biol 2004; 6:1082-1093.

4 Kilpatrick TJ, Bartlett PF. Cloning and growth of multipoten- tial neural precursors: requirements for proliferation and differentiation. Neuron 1993; 10:255-265

5 Palmer TD, Ray J, Gage FH. FGF-2-responsive neuronal progenitors reside in proliferative and quiescent regions of the adult rodent brain. Mol Cell Neurosci 1995; 6:474-486.

6 Alvarez-Buylla A, Garcia-Verdugo JM, Tramontin AD. A unified hypothesis on the lineage of neural stem cells. Nat Rev Neurosci 2001; 2:287-293.

7 Doetsch F, Caille I, Lim DA, et al. Subventricular zone astrocytes are neural stem cells in the adult mammalian brain. Cell 1999; 97:703-716.

8 Eriksson PS, Perfilieva E, Bjork-Eriksson T, et al. Neurogenesis in the adult human hippocampus. Nat Med 1998; 4:13131317.

9 van Praag H, Schinder AF, Christie BR, et al. Functional neurogenesis in the adult hippocampus. Nature 2002; 415:10301034.

10 Okano H, Okano H. The stem cell biology of the central nervous system. J Neurosci Res 2002; 69:698-707.

11 McDonald JW, Liu XZ, Qu Y, et al. Transplanted embryonic stem cells survive, differentiate and promote recovery in injured rat spinal cord. Nat Med 1999; 5:1410-1412.

12 Ogawa Y, Sawamoto K, Miyata T, et al. Transplantation of in vitro-expanded fetal neural progenitor cells results in neurogenesis and functional recovery after spinal cord contusion injury in adult rats. $J$ Neurosci Res 2002; 69:925-933.

13 Iwanami A, Kaneko S, Nakamura M, et al. Transplantation of human neural stem cells for spinal cord injury in primates. $J$ Neurosci Res 2005; 80:182-190.

14 Iwanami A, Yamane J, Katoh $\mathrm{H}$, et al. Establishment of graded spinal cord injury model in a nonhuman primate: the common marmoset. J Neurosci Res 2005; 80:172-181.

15 Takahashi K, Tanabe K, Ohnuki M, et al. Induction of pluripotent stem cells from adult human fibroblasts by defined factors. Cell 2007; 131:861-872.

16 Takahashi K, Yamanaka S. Induction of pluripotent stem cells from mouse embryonic and adult fibroblast cultures by defined factors. Cell 2006; 126:663-676.

$17 \mathrm{Yu}$ J, Vodyanik MA, Smuga-Otto K, et al. Induced pluripotent stem cell lines derived from human somatic cells. Science 2007; 318:1917-1920.

18 Cao QL, Zhang YP, Howard RM, et al. Pluripotent stem cells engrafted into the normal or lesioned adult rat spinal cord are restricted to a glial lineage. Exp Neurol 2001; 167:48-58.

19 Okano H, Ogawa Y, Nakamura M, et al. Transplantation of neural stem cells into the spinal cord after injury. Semin Cell Dev Biol 2003; 14:191-198.

20 Barnabe-Heider F, Frisen J. Stem cells for spinal cord repair. Cell Stem Cell 2008; 3:16-24.

21 Hitoshi S, Seaberg RM, Koscik C, et al. Primitive neural stem cells from the mammalian epiblast differentiate to definitive neural stem cells under the control of Notch signaling. Genes Dev 2004; 18:1806-1811.

22 Miyata T, Kawaguchi A, Okano H, et al Asymmetric inheritance of radial glial fibers by cortical neurons. Neuron 2001; 31:727-741.

23 Noctor SC, Flint AC, Weissman TA, et al. Neurons derived from radial glial cells establish radial units in neocortex. $\mathrm{Na}$ ture 2001; 409:714-720. 
24 Temple S. The development of neural stem cells. Nature 2001; 414:112-117.

25 Okada Y, Matsumoto A, Shimazaki T, et al. Spatiotemporal recapitulation of central nervous system development by murine embryonic stem cell-derived neural stem/progenitor cells. Stem Cells 2008; 2:3086-3098.

26 Bibel M, Richter J, Schrenk K, et al. Differentiation of mouse embryonic stem cells into a defined neuronal lineage. $\mathrm{Nat} \mathrm{Neu}$ rosci 2004; 7:1003-1009.

27 Brederlau A, Correia AS, Anisimov SV, et al. Transplantation of human embryonic stem cell-derived cells to a rat model of Parkinson's disease: effect of In vitro differentiation on graft survival and teratoma formation. Stem Cells 2006; 24:14331440.

28 Keirstead HS, Nistor G, Bernal G, et al. Human embryonic stem cell-derived oligodendrocyte progenitor cell transplants remyelinate and restore locomotion after spinal cord injury. $J$ Neurosci 2005; 25:4694-4705.

29 Yamada M, Tanemura K, Okada S, et al. Electrical stimulation modulates fate determination of differentiating embryonic stem cells. Stem Cells 2007; 25:562-570.

30 Kumagai G, Okada Y, Yamane J, et al. Roles of ES cellderived gliogenic neural stem/progenitor cells in functional recovery after spinal cord injury. PLoS One 2009; 4:e7706

31 Miura K, Okada Y, Aoi T, et al. Variation in the safety of induced pluripotent stem cell lines. Nat Biotechnol 2009; 27:743-745.

32 Tsuji O, Miura K, Okada Y, et al. Therapeutic potential of appropriately evaluated safe-induced pluripotent stem cells for spinal cord injury. Proc Natl Acad Sci USA 2010; 107:1270412709.

33 Okada S, Ishii K, Yamane J, et al. In vivo imaging of engrafted neural stem cells: its application in evaluating the optimal timing of transplantation for spinal cord injury. FASEB J 2005; 19:1839-1841.

34 Nori S, Okada Y, Yasuda A, et al. Grafted human-induced pluripotent stem-cell-derived neurospheres promote motor functional recovery after spinal cord injury in mice. Proc Natl Acad Sci USA 2011; 108:16825-16830.

35 Fujimoto Y, Abematsu M, Falk A, et al. Treatment of a mouse model of spinal cord injury by transplantation of human induced pluripotent stem cell-derived long-term self-renewing neuroepithelial-like stem cells. Stem Cells 2012; 30:11631173 .

36 Kobayashi Y, Okada Y, Itakura G, et al. Pre-evaluated safe human iPSC-derived neural stem cells promote functional re- covery after spinal cord injury in common marmoset without tumorigenicity. PLoS One (in press)

37 Park IH, Zhao R, West JA, et al. Reprogramming of human somatic cells to pluripotency with defined factors. Nature 2008; 451:141-146.

38 Seki T, Yuasa S, Oda M, et al. Generation of induced pluripotent stem cells from human terminally differentiated circulating T cells. Cell Stem Cell 2010; 7:11-14.

39 Hacein-Bey-Abina S, Von Kalle C, Schmidt M, et al. LMO2associated clonal $\mathrm{T}$ cell proliferation in two patients after gene therapy for SCID-X1. Science 2003; 302:415-419.

40 Okano H, Nakamura M, Yoshida K, et al. Steps toward safe cell therapy using induced pluripotent stem cells. Circ Res 2012 (in press).

41 Kaji K, Norrby K, Paca A, et al. Virus-free induction of pluripotency and subsequent excision of reprogramming factors. Nature 2009; 458:771-775.

42 Woltjen K, Michael IP, Mohseni P, et al. piggyBac transposition reprograms fibroblasts to induced pluripotent stem cells. Nature 2009; 458:766-770.

$43 \mathrm{Yu} \mathrm{J,} \mathrm{Hu} \mathrm{K,} \mathrm{Smuga-Otto} \mathrm{K,} \mathrm{et} \mathrm{al.} \mathrm{Human} \mathrm{induced} \mathrm{pluripotent}$ stem cells free of vector and transgene sequences. Science 2009; 324:797-801.

$44 \mathrm{Kim}$ D, Kim CH, Moon J, et al. Generation of human induced pluripotent stem cells by direct delivery of reprogramming proteins. Cell Stem Cell 2009; 4:472-476.

45 Rhee YH, Ko JY, Chang MY, et al. Protein-based human iPS cells efficiently generate functional dopamine neurons and can treat a rat model of Parkinson disease. J Clin Invest 2011; 121:2326-2335.

46 Zhou H, Wu S, Joo JY, et al. Generation of induced pluripotent stem cells using recombinant proteins. Cell Stem Cell 2009; 4:381-384.

47 Jia F, Wilson KD, Sun N, et al. A nonviral minicircle vector for deriving human iPS cells. Nat Methods 2010; 7:197-199.

48 Okita K, Matsumura Y, Sato Y, et al. A more efficient method to generate integration-free human iPS cells. Nat Methods 2010; 8:409-412.

49 Maekawa M, Yamaguchi K, Nakamura T, et al. Direct reprogramming of somatic cells is promoted by maternal transcription factor Glis1. Nature 2011; 474:225-229.

50 Hayashi Y, Chan T, Warashina M, et al. Reduction of N-glycolylneuraminic acid in human iPS cells generated or cultured under feeder- and serum-free defined conditions. PLoS One 2010; 5:e14099. 